\title{
Selective Impairment of the Cerebellar C1 Module Involved in Rat Hind Limb Control Reduces Step-Dependent Modulation of Cutaneous Reflexes
}

\author{
Angelique Pijpers, ${ }^{\star}$ Beerend H. J. Winkelman, ${ }^{\star}$ Robert Bronsing, and Tom J. H. Ruigrok \\ Department of Neuroscience, Erasmus MC Rotterdam, 3000 CA Rotterdam, The Netherlands
}

\begin{abstract}
The cerebellum is divided into multiple parasagittally organized modules, which are thought to represent functional entities. How individual modules participate in cerebellar control of complex movements such as locomotion remains largely unknown. To a large extent, this is caused by the inability to study the contribution of individual modules during locomotion. Because of the architecture of modules, based on narrow, elongated cortical strips that may be discontinuous in the rostrocaudal direction, lesion of a complete module, without affecting neighboring modules, has not been possible. Here, we report on a new method for inducing a selective dysfunction of spatially separated parts of a single module using a small cortical injection of a retrogradely transported neurotoxin, cholera toxin b-subunit-saporin. We show that such a local injection into the $\mathrm{C} 1$ module results in climbing fiber and partial mossy fiber deafferentation of functionally related areas of this module, thereby resulting in a severe impairment of the whole module without affecting neighboring modules. A subsequent functional analysis indicates that such an impairment of the hindlimb part of the $\mathrm{C} 1$ module did not have a significant impact on skilled walking or overall stepping pattern. However, the modulation of cutaneously induced reflexes during stepping was severely diminished. We propose that the $\mathrm{C} 1$ module is specifically involved in the adaptive control of reflexes.
\end{abstract}

Key words: cerebellum; inferior olive; climbing fiber; Purkinje cell; degeneration; locomotion; reflex

\section{Introduction}

Within the uniform structure of the cerebellar cortex, a series of longitudinally arranged modules is recognized. A module consists of one or several functionally related strips, or zones, of Purkinje cells that (1) project to a specific part of the cerebellar nuclei, (2) receive their climbing fibers from a well defined subnucleus of the inferior olive, (3) have specific receptive field characteristics, and (4) differ in their chemical characteristics from adjacent modules (Voogd and Glickstein, 1998). As such, modules are thought to represent functional cerebellar units (Apps and Garwicz, 2005). However, although cerebellar functions are usually described in terms of motor control, coordination, and motor learning, it is not known whether and how individual modules contribute to specific aspects of these functions.

Of course, from the time of Dow and Moruzzi (1958), many cerebellar lesion studies have attributed specific functional roles to different parts of the cerebellar cortex. However, because modules may consist of thin and noncontinuous cortical zones

Received 0ct. 15, 2007; revised Jan. 11, 2008; accepted Jan. 14, 2008.

This work was supported by The Netherlands Organization for Scientific Research, Division of Earth and Life Sciences [NWO-ALW project number 810.37.005 (A.P.), NWO-ALW project number 809.37.007 (R.B.), and NWOVIDI project number 016.048 .306 (B.H.J.W.)] and by the Dutch Ministry of Health, Welfare, and Sports (T.J.H.R.). We thank E. Sabel-Goedknegt, J. van der Burg, and E. Dalm for their expert technical assistance.

*A.P. and B.H.J.W. contributed equally to this work.

Correspondence should be addressed to Tom J. H. Ruigrok, Department of Neuroscience, Erasmus MC Rotterdam,

P.0. Box 2040, 3000 CA Rotterdam, The Netherlands. E-mail: t.ruigrok@erasmusmc.nl.

R. Bronsing's present address: Ratiopharm, $1500 \mathrm{~GB}$ Zaandam, The Netherlands.

D0I:10.1523/JNEUROSCI.4668-07.2008

Copyright $\odot 2008$ Society for Neuroscience $\quad$ 0270-6474/08/282179-11\$15.00/0
(Voogd et al., 2003; Sugihara et al., 2004), it has not been possible to study the function of a complete module without influencing others. Alternative approaches, e.g., interfering with either the output of the modules, i.e., the cerebellar nuclei (Welsh and Harvey, 1991; Koekkoek et al., 2003), or their input [inferior olive (Welsh and Harvey, 1998)], are similarly restricted by the size and location of these nuclei, resulting either in subtotal lesions or in affecting surrounding regions.

Here, we present a new method for inducing dysfunction of elongated and spatially separated parts of a single module. This method is based on the observation that climbing fibers, and to some extent also mossy fibers, collateralize extensively within the confines of a module (Pijpers et al., 2006). After a local cortical injection of a retrogradely transported neurotoxin, consisting of a conjugate of cholera toxin b-subunit and saporin [CTb-S (Llewellyn-Smith et al., 2000)], precerebellar neurons with terminals within the injection site will transport the toxin to their somata, where it will cause their death. Obviously, this will be accompanied by degeneration of their collaterals to spatially distinct, but functionally related, regions of the injected locus. Hence, major parts of the modular entity will become affected (Fig. 1).

We have evaluated this technique and its functional effects on the $\mathrm{C} 1 / \mathrm{C} 3$ hindlimb module of the rat. This module is present as the $\mathrm{C} 1$ zone in the copula pyramidis (COP) and has related regions in the $\mathrm{C} 1$ and $\mathrm{C} 3$ zones of lobules II-V of the anterior lobe (Atkins and Apps, 1997; Voogd et al., 2003; Pijpers et al., 2005). Indeed, we show that a small targeted injection of CTb-S into C1 
of COP results in degeneration within well circumscribed regions of the precerebellar nuclei. Remarkably, the induced impairments produced no or only very mild effects on locomotion per se. However, the step phase-dependent modulation of cutaneously induced reflexes was significantly reduced. We conclude that $\mathrm{CTb}-\mathrm{S}$ is an attractive tool for studying functions of individual cerebellar modules and propose that the $\mathrm{C} 1 / \mathrm{C} 3$ hindlimb module is specifically involved in the adaptive control of reflexes.

\section{Materials and Methods \\ Animals}

Fifty-seven adult, male Wistar rats (weight, 200-300 g) were used in this study. All experiments were performed in accordance with $\mathrm{Na}$ tional Institutes of Health guidelines for the care and use of laboratory animals and with permission from the national committee overseeing animal experiments.

\section{Neurotoxic agent}

As a targeted toxin, CTb-S (Advanced Targeting Systems, San Diego, CA) was used. The ribosome-inactivating protein, saporin (derived from seeds of the plant Saponaria officinalis), cannot be internalized by itself and therefore needs a targeting agent such as CTb. CTb binds to the GM1 ganglioside receptor that is present on most neuronal types and has proven to be a reliable neuronal tracer (Luppi et al., 1995; Ruigrok and Apps, 2007). After internalization and transport to the soma, saporin dissociates from the $\mathrm{CTb}$ and inactivates ribosomes, thereby eventually resulting in cell death. Because at this stage the neurotoxin is no longer bound to a targeting agent, risks of subsequent internalization by surrounding cells will be minimal. Usage and storage protocols were followed according to the manufacturer's details.

\section{Group 1 experiments: anatomical effect of $\mathrm{CTb}-\mathrm{S}$ injections}

Forty animals were used to develop a reliable method to impair selective modules and to monitor histological effects over time. A small quantity of CTb-S (30-50 nl) at full concentration was pressure injected into either the $\mathrm{C} 1$ zone of $\mathrm{COP}$ or paramedian lobule (PMD) of the right cerebellar hemisphere (for surgical procedure, see below). Electrophysiological mapping of climbing fiber receptive fields enabled targeting of the injections at the center of cortical zones (Atkins and Apps, 1997). Animals were killed at various time intervals after injection to establish a time course of the resultant CTb labeling. Neuronal degeneration was assessed by a suppressed silver degeneration technique and cell counts of the dorsal accessory olive (see below for further details). Within this group, two rats received an injection of $50 \mathrm{nl}$ of saline in the $\mathrm{C} 1$ zone of COP.

\section{Group 2 experiments: behavioral effect of CTb-S injections}

The behavioral effect of CTb-S injection was assessed in 11 animals by evaluating their skilled walking in the ladder rung-walking test as described by Metz and Whishaw (2002). In this test, the number of misplaced hindpaw steps was scored when rats repeatedly cross a horizontal ladder with changing patterns of rungs. A few changes were made to the original testing paradigm. First, the animals had to cross the ladder in 10 nucleus; $\mathrm{PO}$, principal olive.
$\mathrm{B}$

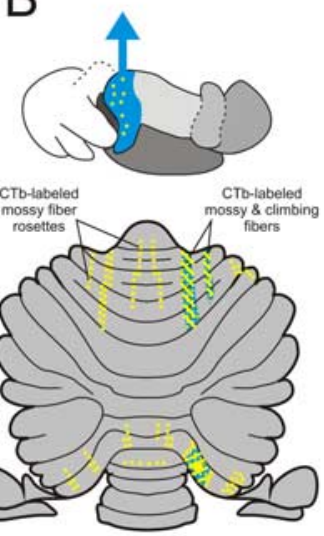

C
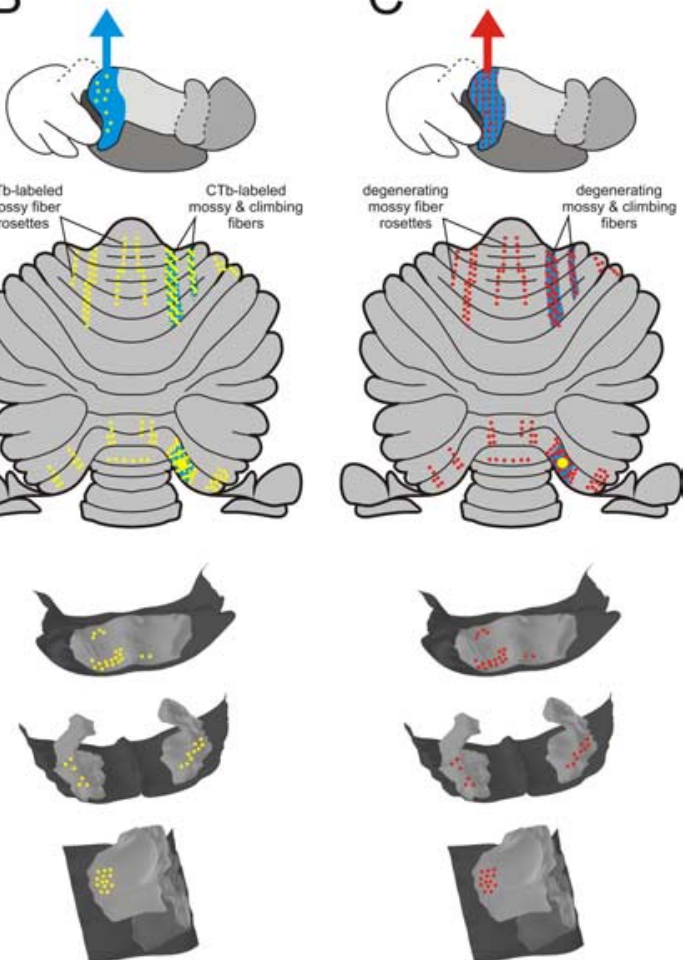

Distribution of CTb-labeled neurons
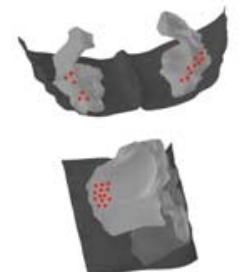

Degeneration of CTb-labeled neurons by saporin intoxication

Figure 1. Diagrams explaining rationale of CTb-S injection within the C1 zone of COP. A, Top, Dorsal view of the cerebellar nuclei. Middle, Schematic diagram of the unfolded cerebellar cortex (Voogd et al., 2003). Bottom, Three three-dimensional the function of the related module, all blue areas would need to be affected simultaneously. An injection with CTb-S in the center 政 Pijpers and Ruigrok, 2006). Also note labeled nucleocortical neurons (top). The distribution of climbing fiber collaterals of these cells involves the $\mathrm{C} 1$ and $\mathrm{C} 3$ zones in the anterior lobe (Voogd et al., 2003; Pijpers et al., 2006) and is shown by yellow hatching, whereas the approximate distribution of mossy fiber collaterals is indicated by small yellow dots (Pijpers et al., 2006). C, After the sorin induces cell death of the (Tb-S-labeled precerebellar neurons (red dots), all their collaterals will also degenerate (small red cell groups; LCN, lateral cerebellar nucleus; MA0, medial accessory olive; $M C N$, medial cerebellar nucleus; PIN, posterior interposed

rather than five different irregular patterns. Second, a mirror was placed at an angle behind the ladder, enabling videotaping both sides of the animal in one shot. After $2 \mathrm{~d}$ of initial training, to get acclimated to walking the apparatus in regular patterns, and 3 subsequent days of testing, the animals underwent surgery. The second day after surgery, testing was resumed for $10 \mathrm{~d}$. In this group, seven animals were injected with conjugate into the $\mathrm{C} 1$-hindlimb receiving area of the COP in the right cerebellar hemisphere. Two animals with $\mathrm{C} 1$ saline injections were used as controls. In the remaining two cases, a CTb-S injection was made into the hindlimb region of the right motor cortex. Details on surgery, video analysis, and statistics are presented below.

\section{Group 3 experiments: physiological effect of CTb-S injections}

To evaluate the effects of CTb-S injections on locomotion and on induced reflexes during locomotion, a third group of six animals underwent daily sessions for $\sim 2$ weeks, in which they learned to walk on a walking belt for at least $1 \mathrm{~min}$ at constant speed $(\sim 10 \mathrm{~m} / \mathrm{min})$. Subsequently, they underwent instrumentation with EMG electrodes in the left 
biceps femoris muscle (BF) and subcutaneous placement of stimulation electrodes near the left lateral malleolus (for surgery details, see section below). Four of these animals received a CTb-S injection into C1-COP of the left cerebellar hemisphere, and two sham-operated cases were injected with saline at the time of instrumentation. Surgical and ethical constrictions did not allow us to collect EMG data before and after injections.

After a $2 \mathrm{~d}$ recovery period, rats were monitored in daily recording sessions for a period of up to 2 weeks (Bronsing et al., 2005). These sessions started by determining stimulation thresholds for the subcutaneously placed electrodes at rest. This was followed by several minutes of nonstimulated (normal) walking. Subsequently, the BF-EMG during a minimum of $121 \mathrm{~min}$ bouts of locomotion was recorded while the subcutaneous leads were randomly stimulated at 1.5 times threshold (during stimulated walking). Single stimulus pulses of $0.1 \mathrm{~ms}$ were used and had thresholds between 80 and $250 \mu \mathrm{A}$ and a latency of $\sim 10 \mathrm{~ms}$. Such a stimulation paradigm would be in accordance with $A \beta$ triggered reflex responses (Woolf and Swett, 1984). Because of subcutaneous location of the electrodes, participation of some skeletal mechanoreceptors cannot be excluded. Stimulation thresholds and responses were quite constant between various days. Randomly timed pulses were generated using two Gaussian clocks programmed in the stimulus Sequencer of a 1401 Plus (Cambridge Electronic Design, Cambridge, UK) and were delivered at an average frequency of $0.4 \mathrm{~Hz}$ and with a minimum interstimulus interval of $0.5 \mathrm{~s}$. These pulses triggered a PG4000 digital pulse generator and isolated stimulation unit (both Neuro Data Instruments, New York, NY). EMG signals were amplified $(5000 \times)$ and bandpass filtered $(30 \mathrm{~Hz}$ to $10 \mathrm{kHz}$; Axon CyberAmp 380; Molecular Devices, Sunnyvale, CA). A humbug device (Quest Scientific, North Vancouver, British Columbia, Canada) was used to eliminate $50 \mathrm{~Hz}$ electrical interference. EMG signals were sampled with the 1401 Plus at $8.333 \mathrm{kHz}$ and stored for off-line analysis using Spike 2 data acquisition and analysis software (Cambridge Electronic Design).

\section{Surgical procedures}

In the experimental groups 1 and 2 , animals were initially anesthetized by an intraperitoneal injection of $60 \mathrm{mg} / \mathrm{kg}$ pentobarbital (Nembutal; Ceva Sante Animale, Maassluis, The Netherlands). In addition, supplementary doses were given intraperitoneally to maintain deep levels of anesthesia. This anesthetic was chosen because it allows the identification of cortical zones by extracellular recording of evoked climbing fiber field potentials while reducing mossy fiber responses (Atkins and Apps, 1997). The rats of experimental group 3 were anesthetized with isoflurane (5\% initially, $0.5-2 \%$ maintenance in $0.5 \mathrm{~L} / \mathrm{min}$ oxygen and $1 \mathrm{~L} / \mathrm{min}$ nitrous oxide). In all cases, the depth of anesthesia was monitored by absence of the pinch withdrawal reflex and loss of muscle tone. Body temperature was maintained and regulated within physiological limits with a thermostatically controlled heating pad $\left(37 \pm 1^{\circ} \mathrm{C}\right)$.

Animals were subsequently placed in a Kopf stereotaxic head holder, and the posterior cerebellum was accessed as described earlier (Pijpers et al., 2006). In group 1 and 2 animals, evoked field potentials were recorded on the right cerebellar surface with glass-coated tungsten microelectrodes (tip, $\sim 50 \mu \mathrm{m}$; impedance, $50-140 \mathrm{k} \Omega$ ) as the result of brief $(0.1 \mathrm{~ms})$ electrical percutaneous stimulation of the ipsilateral or contralateral forelimb or hindlimb with pairs of needle electrodes (Atkins and Apps, 1997). In this way, the location of the C1 zone of the COP or PMD was identified by their relatively short latency field potential that was triggered from ipsilateral hindlimb or ipsilateral forelimb stimulation, respectively. Microinjections were subsequently made at the site where maximum responses were evoked, which was usually at the center of the identified $\mathrm{C} 1$ zone. Because the location of especially the $\mathrm{C} 1$ zone of the COP proved to be very reproducible, no electrophysiological verification was considered to be required for the group 3 experiments.

Injections of CTb-S or saline were made with a custom-made pressure device attached to filament-containing glass micropipettes (tip diameter, $8-12 \mu \mathrm{m}$ ) (Ruigrok and Apps, 2007). Micropipettes were advanced perpendicular to the cerebellar surface with a micromanipulator, and injections were placed $200-300 \mu \mathrm{m}$ below the cerebellar surface. The pipette was left in situ for $10 \mathrm{~min}$, after which it was withdrawn and the surface was flushed with saline. Subsequently, the overlying muscle and skin layers were sutured, and the animal was allowed to recover for group 1 and 2 experiments.

For group 3 experiments, surgery proceeded with the implantation of custom-prepared bipolar EMG electrodes, made of Teflon-isolated multistrand stainless steel wire (diameter, $150 \mu \mathrm{m}$; exposed tip length, $\sim 1$ $\mathrm{mm}$; Advent Research Materials, Oxford, UK). Through a small incision $(2 \mathrm{~cm})$ of the skin over the lateral thigh region, three of these electrodes were implanted along the posterior part of the BF of the left hindlimb just below the muscle fascia, to which they were secured by a single suture (6.0; Ethicon, Piscataway, NJ). In addition, two similar wires serving as stimulation electrodes were implanted on either side of the left lateral malleolus by maneuvering a cutoff injection needle $(0.8 \times 40 \mathrm{~mm})$, in which the proximal end of the wire was clamped, from the ankle region to the opened thigh region. Subsequently, needle and wire were pulled until the stripped $(2 \mathrm{~mm})$ distal part of the wire, which was widened with a small drop of Araldite to $\sim 1 \mathrm{~mm}$, could be just pushed under the skin. The Araldite drop prevented subcutaneous movement of the stimulation electrode. The leads were further secured to subcutaneous fascia in the thigh region. All leads were looped to allow sufficient slack and were tunneled subcutaneously to the incision in the neck. Here the wires were looped again and attached to a connector, which was secured in a pedestal of dental cement, which was fixed to the skull with screws (Bronsing et al., 2005). All skin incisions were carefully closed with single sutures, and the animals were allowed to recover.

In all cases, postoperative analgesia was provided by subcutaneous administration of buprenorphine $(0.05-0.1 \mathrm{mg} / \mathrm{kg}$; Temgesic; ScheringPlough, Utrecht, The Netherlands). Animals were monitored daily for signs of stress or discomfort but in all cases recovered uneventfully.

\section{Histology}

At the end of each experiment, animals were killed by means of transcardial perfusion under deep barbiturate anesthesia $(240 \mathrm{mg} / \mathrm{kg}$, i.p.; Nembutal). An initial flush with $500 \mathrm{ml}$ of $0.9 \%$ saline was followed by 1000 $\mathrm{ml}$ of $4 \%$ paraformaldehyde and $4 \%$ sucrose in $0.05 \mathrm{~m}$ phosphate buffer (PB), $\mathrm{pH}$ 7.2-7.4. The brain was postfixed for $3 \mathrm{~h}$, rinsed, and stored overnight in $0.05 \mathrm{M} \mathrm{PB}$ with $10 \%$ sucrose (at $4^{\circ} \mathrm{C}$ ) and embedded in $11 \%$ gelatin, $10 \%$ sucrose. Blocks were hardened in a $10 \%$ formalin, $30 \%$ sucrose solution for $3 \mathrm{~h}$ and, after further cryoprotection in PB with $30 \%$ sucrose, they were sectioned transversally at $40 \mu \mathrm{m}$ on a freezing microtome and serially collected in PB in eight glass vials. Selected vials were processed free-floating as described earlier either for CTb histochemistry (Ruigrok et al., 1995; Ruigrok and Apps, 2007) or for silver degeneration (Haasdijk et al., 2002).

Microphotographs were acquired with a Leica (Wetzlar, Germany) DMR microscope equipped with a digital camera (Leica DC-300 at $2088 \times 1552$ pixels). Photo panels were constructed in CorelDraw 11.0, after correction for brightness and contrast in Corel Photopaint 11.0 (Corel, Ottawa, Ontario, Canada). Neuron counts of the dorsal accessory olive (DAO) were obtained by plotting a one-in-four series of transverse sections (160 $\mu \mathrm{m}$ interval) using an Olympus (Tokyo, Japan) microscope fitted with a Lucivid miniature monitor and Neurolucida software (MicroBrightField, Colchester, VT). The outlines of each inferior olive were traced with a $2.5 \times$ objective and, subsequently, individual neurons in the DAO were indicated using a $20 \times$ objective and the motorized stage scan option of the plot program. With the automated cell count option, the number of olivary neurons within $100-\mu \mathrm{m}$-wide bins (plotted perpendicular to the main direction of the DAO) was determined for consecutive sections. Only neuronal contours within the contours of the DAO and with a clearly discernible nucleus were counted. From these data, color-coded and interpolated density profiles of the DAO were constructed using standard MATLAB routines (The MathWorks, Natick, MA) (Pijpers and Ruigrok, 2006). Abercrombie's correction factor was used to obtain an estimate of the total number neurons in the DAO.

\section{Video analysis}

The skilled walking tests of group 2 rats were videotaped with a Sony (Tokyo, Japan) Digital Handycam (DCR-PC8E) at 30 frames/s. In each case, the number of errors in foot placement accuracy was counted for 
both hindlimbs. The video recordings were analyzed at 0.2 times normal speed, and the number of observed foot faults, defined as a slip or a total miss, was determined for each hindlimb per test day per rat. No learning/ improvement was noted before or after injection. Therefore, for each case, the data before and after injection were pooled, and the percentage of scored mistakes was determined from which a group average \pm SEM before and after injection was calculated for the left and right hindlimbs, respectively.

\section{EMG analysis}

EMG analysis was performed off-line and consisted of four steps.

(1) Data preprocessing. Custom MATLAB routines were used to import and analyze the stimulation times and EMG data. The raw EMG signal was preprocessed by masking stimulation artifacts for a period of 4 $\mathrm{ms}$ from the onset of stimulation followed by digital bandpass filtering $(100-3000 \mathrm{~Hz})$ using a fifth-order Butterworth filter. The EMG amplitude was computed by root-mean-square (RMS) rectification using a 20 ms window.

(2) Step identification and standardization. BF EMG activity of a single step cycle was partitioned into three distinct phases: a burst of activity (BA), a silent period (SP), and a period of sustained activity (SA). The onset of BA was chosen as the start and end of a step cycle (Bronsing et al., 2005). Cursors delineating these step phases were placed on the basis of visual inspection of the EMG and EMG amplitude signals. Occasional occurring step cycles with a duration shorter than $0.4 \mathrm{~s}$ or longer than $1.4 \mathrm{~s}$ were discarded. To allow averaging and comparison of EMG activity from steps with different lengths, EMG amplitude data were extracted, and step duration was normalized to $100 \%$. Accordingly, the duration of all step cycle phases was normalized to step cycle fractions. Ideally, EMG activity of all steps at a particular relative time point within a step cycle should all belong to the same step cycle phase (BA, SP, or SA). Therefore, a standard step cycle was computed by averaging the step cycle fractions over all rats and all days $(\mathrm{BA}=9.83 \%$; $\mathrm{SP}=13.08 \%$; $\mathrm{SA}=77.09 \%)$. Subsequently, EMG amplitude data from all steps were time rescaled so that the step cycle fractions of each step equaled the standard step. This time rescaling was achieved by calculating the projection of the integrated step fractions of the standard step onto the integrated step fractions of each actual step using piecewise cubic spline interpolation of 1000 linearly spaced time bins, followed by linear interpolation of the EMG amplitude data onto the 1000 time-rescaled bins. This procedure produced a gradual deformation of the time axis and on average equal time per bin.

(3) EMG normalization. Because EMG data from different rats vary in gain and noise levels, EMG amplitude data per rat for each day were normalized to the range of the average step cycle EMG, computed for each rat per day from all steps not including stimulation, and the minimum average step cycle EMG was subtracted.

(4) Comparing reflex size. The height of the normalized EMG amplitude $15 \mathrm{~ms}$ after a stimulus (corresponding to the RMS activity from 5 to $25 \mathrm{~ms}$ after stimulation) was used to obtain a measure of the reflex magnitude. The value of the previously computed and normalized average step cycle EMG at the same point in the step cycle was subtracted from the reflex magnitudes to obtain the net reflex magnitudes, which in turn were averaged in 5\% step cycle bins for each day or in step phase bins over all days. Error bars indicate the SEM. Between the sham and lesion group, the mean reflex response per step cycle bin was compared using a two-sample Student's $t$ test and Bonferroni correction for multiple comparisons ( $p=0.0005$ for the per day comparison; $p=0.015$ for the step cycle phase comparison).

\section{Results}

\section{A local CTb-S injection affects modular integrity}

Initial experiments were designed to chart the effects of a single local injection of CTb-S in the cerebellar cortex (group 1 experiments). In order for the CTb-S to have the desired effect of affecting related regions at different parts of the cerebellar cortex, it was necessary to show that, apart from degeneration of the neurons within the injection site, the afferent systems that reach a specific injection site also degenerate. Because the modular relation between areas of the inferior olive, cerebellar nuclei, and Purkinje cell zones are well known and provide identification of the targeted cerebellar module (Ruigrok, 1997; Voogd et al., 2003; Sugihara et al., 2004; Pijpers et al., 2005), we focus our description on these areas.

Twenty-four hours after CTb-S injection, the injection sites were clearly demarcated by CTb immunohistochemistry (Fig. $2 A 1, B 1)$ and had a diameter of $300-500 \mu \mathrm{m}$, which is similar to that of iontophoretic delivery of unconjugated CTb (Ruigrok et al., 1995; Ruigrok and Apps, 2007). No degeneration of cortical structures could be observed within or around the injection site at this time with either thionin or silver degeneration staining. Evidence for uptake and transport of the conjugate, however, was observed because CTb immunohistochemistry revealed many retrogradely labeled neurons at various places in the brainstem, such as the pontine nuclei, lateral reticular nucleus, and inferior olive (IO), as well as within the cerebellar nuclei. In addition, neurons within the injection site had incorporated CTb-S, as evidenced by anterograde labeling of terminal arborizations of Purkinje cell efferents within the cerebellar nuclei. Figure $2 \mathrm{~A}$ shows typical results from a CTb-S injection in the $\mathrm{C} 1$ region of COP. Labeled IO neurons were confined to the caudolateral aspect of the ventral fold of the dorsal accessory olive (vfDAO), whereas CTb-labeled terminals were noted medially within the anterior interposed nucleus (AIN). In contrast, CTb-S injections within the $\mathrm{Cl}$ zone in the $\mathrm{PMD}$ resulted in CTb-labeled neurons that were restricted to the medial aspect of the vfDAO, and labeled Purkinje cell terminals were positioned within the central part of the AIN (Fig. 2B). These locations are in perfect agreement with earlier data on topography of olivocorticonuclear connections and show that the tracer characteristics of CTb-S are very similar to those of unconjugated CTb (Pardoe and Apps, 2002; Voogd et al., 2003; Sugihara et al., 2004; Pijpers et al., 2005; Pijpers and Ruigrok, 2006). Note that both types of injections also resulted in retrograde labeling of nucleocortical neurons, which were mostly confined to regions that also harbored anterograde terminal labeling (Provini et al., 1998) (Fig. 2A2,A3,B2,B3).

Signs of cortical degeneration were only noted with survival times of $2 \mathrm{~d}$ or more (Fig. 2C1,D1). For example, argyrophilic degeneration became clearly visible at survival times of at least $4 \mathrm{~d}$. Silver deposits were most prominent over degenerating fibers as was seen in the medial and central AIN for C1-COP and C1PMD injections, respectively (Fig. 2C3,D3), but was less robustly noted within the corresponding areas of the IO (Fig. 2C5,D5). At this time, $\mathrm{CTb}$ immunohistochemistry also resulted in less intense labeling of the involved IO regions (Fig. 2, compare C6 and $D 6$ with $A 5$ and B5). Moreover, the labeled neuronal profiles were small without clearly identifiable nuclei, and proliferation of glial structures was noted in the neuropil surrounding them (Fig. $2 C 6, D 6)$. At the same time, CTb-labeled terminals and neurons were no longer observed within the appropriate AIN regions that now contained silver deposits, implying major degeneration of neuronal structures (Fig. 2C2,D2). After a survival time of $10 \mathrm{~d}$ or longer, apart from faint labeling near the injection site, no $\mathrm{CTb}$ label could be recovered.

Figure $2 E$ shows argyrophilic staining of degenerated structures in another case with a CTb-S injection that was targeted at C1-COP and surviving for $21 \mathrm{~d}$. Apart from silver deposits within and around the injection site that could be followed to the medial half of the AIN (data not shown, but similar to Fig. 2C3), degeneration was noted in all cerebellar peduncles (Fig. 2E1). Silver staining in the inferior cerebellar peduncle could be traced to the 

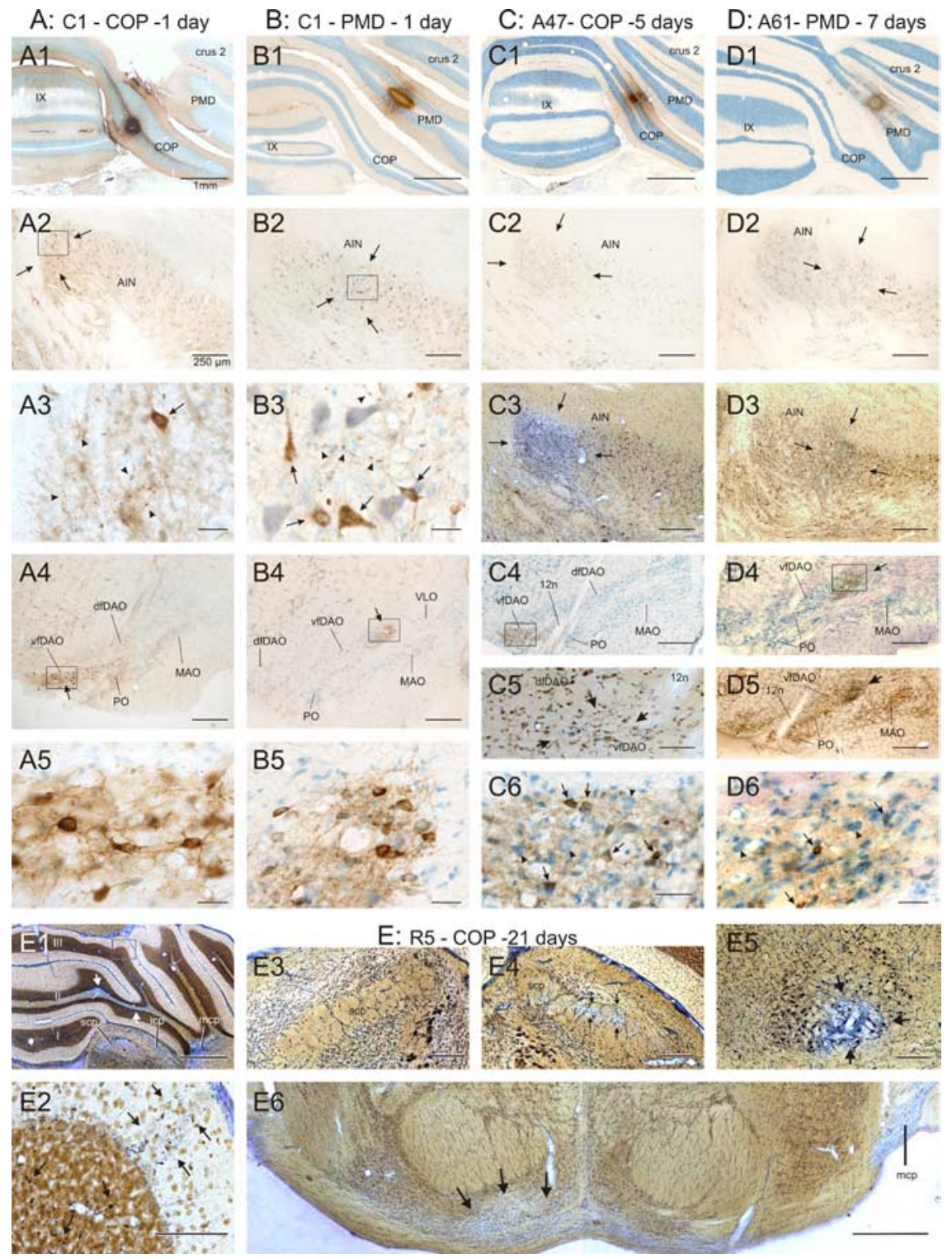

Figure 2. Histological effect of CTb-S injection after various survival times. $A$, Case A53 was injected in the C 1 zone of COP and was killed after $1 \mathrm{~d}$. A1, The injection site was visualized with CTb immunocytochemistry. Virtually no degeneration was observed at this stage. A2, A3, Anterograde transport of the CTb-S by Purkinje cell axons was indicated by labeling of fibers with varicosities (A3, arrowheads) in the medial AIN (A2, arrows). A3-A5, Retrograde transport resulted in labeling of several nucleocortical projecting neurons $(\boldsymbol{A} 3$, arrow) and of olivary neurons in the caudolateral part of the vfDA0 $(\boldsymbol{A 4}, \boldsymbol{A 5}) . \boldsymbol{A 3}, \boldsymbol{A} \boldsymbol{5}$, Enlargements of the boxed areas in $\boldsymbol{A 2}$ and $\boldsymbol{A 4}$, respectively. $\boldsymbol{B}$, Similar to $\boldsymbol{A}$, but now for case $A 58$ with a CTb-S injection in the C1 region of PMD (B1). Note that anterograde and retrograde labeling (arrowheads and arrows, respectively, in $B 3$ ) is located in a more central region of the AIN (B2), whereas retrograde labeling within the vfDA0 was restricted to its medial aspect $(\boldsymbol{B} 4, \boldsymbol{B} 5)$. $\boldsymbol{C}$, Injection similar to $\boldsymbol{A}$, but now, after a survival time of $5 \mathrm{~d}$, CTb histochemistry hardly revealed labeling of neurons or fibers within the AIN (C2, C3). C3, Reduced silver staining of an adjacent section now showed abundant argyrophilic degeneration confined to the medial AIN. (4-C6, In the inferior olive, both CTb labeling (in the caudolateral vfDAO; $\mathbf{C 4}, \mathbf{C 6})$ and silver degeneration in a corresponding region of an adjacent section (C5, arrows) were noted. $\mathbf{C 6}$, Enlargement of the boxed area of $\mathbf{C}$. Note that the size of the CTb-labeled somata is reduced compared with $\boldsymbol{A} \boldsymbol{5}$ and $\boldsymbol{B} \boldsymbol{5}$ or with the nonlabeled neuronal profiles (arrowheads). $\boldsymbol{D}$, Similar set of photographs as in $\boldsymbol{C}$, but now for case $A 61$ with an injection of C1 in PMD and a survival time of $7 \mathrm{~d}$. Note failure of CTb labeling in AIN (D2) and scant labeling within the inferior olive $(\mathbf{D 4}, \mathbf{D 6})$. Silver degeneration within the central AIN (D3) and medial vfDA0 (D5) indicates degeneration of neuronal structures. D6, Enlargement of the boxed area of D4 showing, apart from neuropil labeling, some small CTb-labeled neuronal profiles [arrows; compare with unlabeled profiles (arrowheads)]. $\boldsymbol{E}$, Aspects of argyrophilic degeneration in case R5 with an injection of $\mathrm{C} 1$ in COP and a survival time of $21 \mathrm{~d}$. E1, White matter degeneration within the anterior lobe and all three cerebellar peduncles. E2, Enlargement of the boxed area in E1, showing degeneration in granular and molecular layers (arrows) at an area corresponding to C1 (Pijpers et al., 2005). E4, Degeneration of nucleobulbar fibers in the ipsilateral superior cerebellar peduncle (scp; arrows) but not in the contralateral $\operatorname{scp}(\boldsymbol{E} 3)$. $\boldsymbol{E 5}$, Degeneration of nucleobulbar fibers in ventral part of contralateral red nucleus (arrows). E6, Degeneration of axons and neurons within the caudal part of the basilar pontine nuclei and ipsilateral medial cerebellar peduncle (mcp). Scale bars: A1-E1, E6, $1 \mathrm{~mm}$; A2-D2, C3-E3, A4-E4, C5-E5, $250 \mu \mathrm{m} ; \mathbf{E 2}, 100 \mu \mathrm{m} ; \mathbf{A 3}, \mathbf{A 5}, \mathbf{B 3}, \mathbf{B 5}, \mathbf{B 6}, \mathbf{D 6}, 25 \mu \mathrm{m}$. I-X, Cerebellar lobules I-X; 12n, hypoglossal nerve; crus 2, cerebellar lobule crus 2; icp, inferior cerebellar peduncle; $M A 0$, medial accessory olive; $P 0$, principal olive. contralateral vfDAO and the ipsilateral lateral reticular nucleus (data not shown), whereas abundant degeneration of precerebellar neurons in the caudal pontine nuclei (Pijpers and Ruigrok, 2006) resulted in axonal degeneration of the middle cerebellar peduncle (Fig. 2E6). Many degenerating fibers were also found in the dorsal region of the central part of the ipsilateral superior cerebellar peduncle (Fig. $2 E 3, E 4)$. This region is known to specifically contain the efferent fibers from the AIN (Haroian et al., 1981). We presume that these degenerating fibers, which could be followed to a field of terminal degeneration in the ventral part of the contralateral magnocellular red nucleus (Fig. 2 E5), reflect bulbar projection fibers from AIN neurons that had incorporated the conjugate via nucleocortical collaterals (Tolbert et al., 1978).

Evidence that the neurons of origin of the climbing and mossy fibers to the C1COP injection site had expired was further provided by the observed silver staining of fibers in the paravermal white matter of lobules II-V of the anterior lobe (Fig. 2E1). These degenerating fibers can only reflect the collaterals of climbing and mossy fibers that possessed terminals within the injection site. In accordance with the bilateral nature of the collateralization of the mossy fiber system, some fibers were also noted at the contralateral side. Unfortunately, only relatively sparse terminal labeling was noted in the granular and molecular layer of the ipsilateral paravermis of lobules II-V (Fig. 2E2), which did not justify the abundance of collateral projections of both climbing and mossy fibers from the same cortical regions seen when using unconjugated $\mathrm{CTb}$ as a tracer (Voogd et al., 2003; Pijpers et al., 2006). The difficulty of demonstrating robust terminal degeneration of climbing fibers has, however, been noted repeatedly in classic literature (Desclin, 1974).

Therefore, to verify the modular effect of small, localized lobular injections with CTb-S, it was essential to chart the degeneration of olivary neurons in the appropriate places. Hence, neuron counts of both the vfDAO and dorsal fold of dorsal accessory olive ( $\mathrm{dfDAO}$ ) were made bilaterally for four animals with an injection into the $\mathrm{C} 1$ zone of the right COP, two animals with a saline injection at the same place, and an additional case with a CTb-S injection targeted to C1 of PMD (case 61). The results are presented in Figure 3 in colorcoded diagrams reflecting the density of neuronal cell bodies in flattened and unfolded plots of the contralateral and ipsi- 


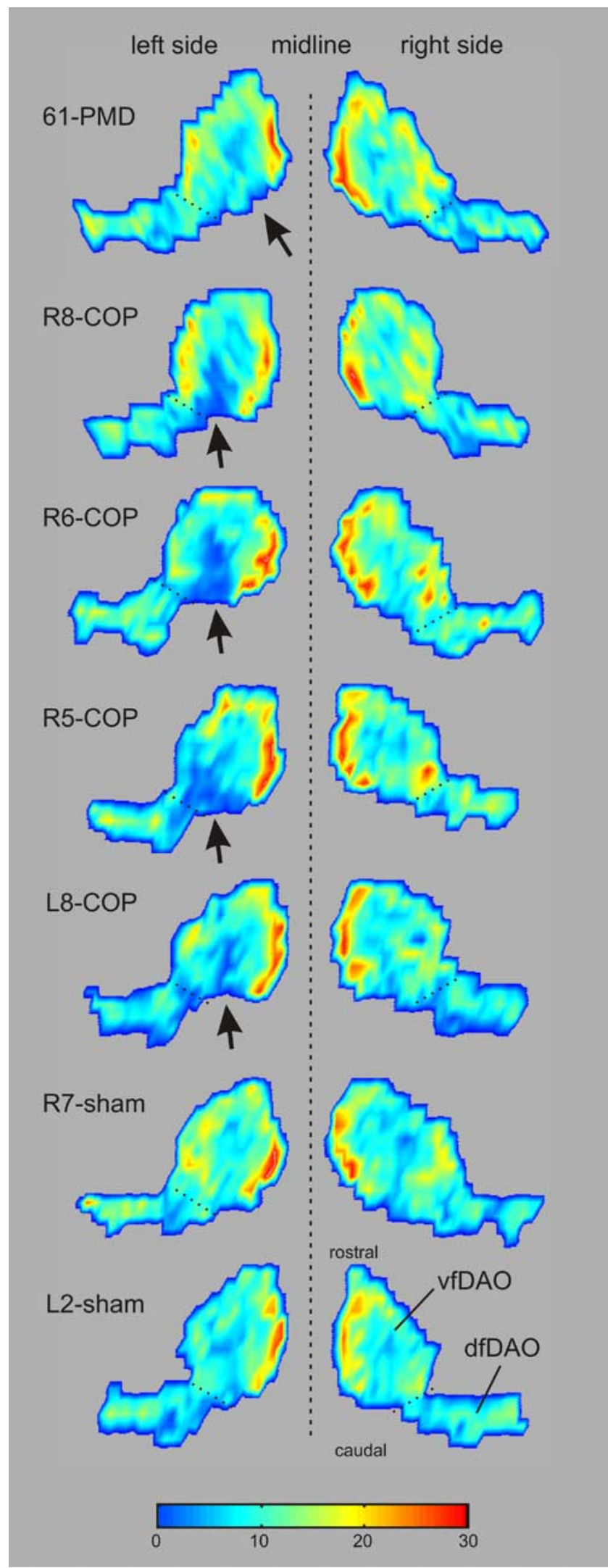

Figure 3. Color-coded density plots of neuronal profiles in the flattened and unfolded dorsal accessory olives after CTb-S or saline (sham) injection in the right COP of five animals (survival times, 8-21 d). For comparison, a case with a 11 injection of right PMD (case A61; survival time, $7 \mathrm{~d}$ ) was included. Note that neurons are unevenly distributed throughout the ipsilateral dorsal accessory olive (right side) and display the highest cell densities at the medial and lateral aspects of the nucleus. Occasional small patches with few cells in the center of the nucleus were lateral DAO. These diagrams show that CTb-S injection into $\mathrm{C} 1-$ COP results in markedly lower densities of neurons within the caudolateral aspect of the contralateral vfDAO. In several animals, virtually no neuronal contours were noted within this region (e.g., cases R5, R6). Densities of neurons in the other regions of the DAO were similar to those seen at the side ipsilateral to the injection. The contralateral DAO contained on average $85 \%$ (range, $80.1-89.4 \% ; n=5$ ) of the number of neurons found in the ipsilateral DAO. This equals degeneration of $\sim 900$ olivary neurons in the whole DAO, which would be specifically located in the caudolateral part of vfDAO. This area has been shown to be involved in hindlimb control (Atkins and Apps, 1997) and to collateralize extensively to the $\mathrm{C} 1$ and $\mathrm{C} 3$ zones of the anterior lobe (Voogd et al., 2003; Pijpers et al., 2006). Injection into the C1-PMD zone (responsive to ipsilateral forelimb stimulation) resulted in a reduction of neuronal density of the caudomedialmost part of the vfDAO, which is related to $\mathrm{C} 1$ aspects of the forelimb and collateralizes to simple lobule (Atkins and Apps, 1997; Pardoe and Apps, 2002; Pijpers et al., 2006). Cortical injections of saline did not result in changes in cell densities within the DAO (Fig. 3, cases R7 and L2).

We conclude that transport of CTb-S is perfectly in line with previously conducted studies on the modular connectivity of the rat cerebellum (Atkins and Apps, 1997; Voogd et al., 2003; Voogd and Ruigrok, 2004; Pijpers et al., 2005; Pijpers and Ruigrok, 2006). The well circumscribed olivary area with initial CTb labeling followed by silver degeneration and eventual cell loss indicates that a single and specific cerebellar module can be targeted, resulting in subsequent deafferentation of a major part of the climbing fiber input to the whole module.

\section{Impairment of C1-hindlimb module has no impact on skilled walking}

Purkinje cells of the $\mathrm{C} 1$ zone of the COP receive climbing fibers that respond to ipsilateral hindlimb stimulation (Atkins and Apps, 1997). Moreover, mossy fiber rosettes that originate from the lumbar spinal cord as well as from hindlimb-related regions of the pontine nuclei also reach this part of the cerebellum in abundant numbers (Gravel and Hawkes, 1990; Pijpers and Ruigrok, 2006). This suggests that impairment of the related module is likely to specifically affect control of hindlimb muscles. In a second group of experiments, we have tested skilled walking performance in a ladder rung-walking test (Metz and Whishaw, 2002). Locomotion skills of animals with a CTb-S injection into the right C1-COP were compared with the performance of animals with a saline injection into the same region, as well as with two cases with a CTb-S injection into the hindlimb region of the right motor cortex. The latter injections resulted in degeneration of fibers in the corpus callosum, the ipsilateral internal capsule, caudate-putamen, pontine nuclei, pyramidal tract, and contralateral dorsal corticospinal tract that could be followed to the lumbar spinal cord, as well as in neuronal cell loss within the ventrolateral thalamus (data not shown). Histology of the cerebella of the C1-COP-injected animals all revealed degeneration of

\section{$\leftarrow$}

caused by traversing bundles of the hypoglossal nerve. Note that the CTb-S injections in COP resulted in diminished density of neurons in the caudolateral part of the left vfDAO (arrows). No such change was observed after saline injections (R7-sham and L2-sham; bottom panels). PMD injection resulted in a decrease of neurons in the caudomedial part of the vfDAO (arrow in top panel; 61-PMD). Dotted lines mark approximate position of the "folding" line between dfDA0 and vfDAO (Ruigrok, 2004). The color scale indicates the number of neurons per $100-\mu \mathrm{m}$-wide bin. 
A

Left hind limb

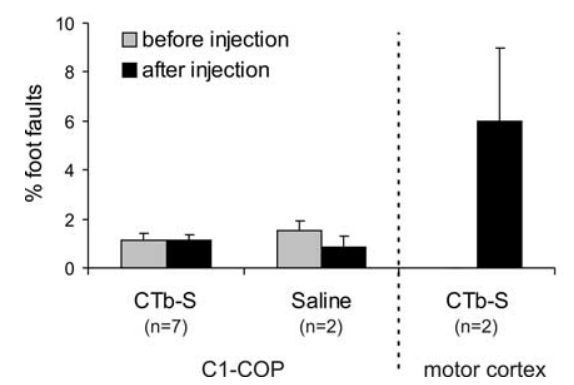

B

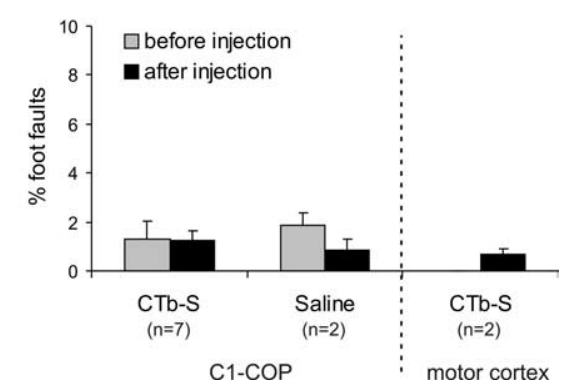

Figure 4. Evaluation of skilled walking before (gray bars) and after (black bars) injection of (Tb-S or saline on the ladder rung-walking test. $\boldsymbol{A}$, Data pertaining to the left hindlimb. $\boldsymbol{B}$, Data pertaining to the right hindlimb. Data of consecutive days before and after surgery were pooled for each case. The CTb-S group involved seven animals with injections in the right C1-COP, in which a total of 3696 steps were examined before and 12,496 steps after injection. In the saline group (injection in right (1-COP), 1755 steps before and 3316 steps after injection were examined in two animals. CTb-S was injected in the hindlimb-related area of the right motor cortex in two animals, and 2166 steps were examined after injection only. Approximately the same number of steps, evenly distributed over the left and right hindlimb, was studied for every case. Bars indicate the group average percentage + SEM of steps with a misplacement (slip or missed bar). The only marked postinjection decline of walking proficiency (i.e., an increase in number of foot faults of specifically the left hindlimb) was seen after injection of (Tb-S into the hindlimb-related area of the right motor cortex.

paravermal parts of the COP and displayed signs of neuronal degeneration of olivary neurons within the caudolateral parts of the contralateral vfDAO comparable with those described above.

Figure 4 shows the average percentage of misplaced foot steps or slips collected from the ladder rung-walking apparatus in a series of 3 test days before and in up to 11 test days after the CTb-S injection. Injections into the right cerebellar cortex are expected to predominantly affect the ipsilateral side, whereas injections into the motor cortex are expected to have an effect on the contralateral limbs. However, our results show that $\mathrm{CTb}-\mathrm{S}$ injection into $\mathrm{C} 1$ of the COP did not result in an increase of stepping errors in either hindlimb. A significant increase in the number of foot faults was only seen after CTb-S injection of the motor cortex, where the average number of induced errors contralateral to the injected side was similar to that reported by Metz and Whishaw (2002) after mechanical lesions of the motor cortex or the pyramidal tracts. Therefore, we conclude that, unexpectedly, CTb-S injection of C1-COP does not result in a conspicuous deterioration of skilled walking.

\section{Impairment of C1-hindlimb module reduces step phase- dependent modulation of reflexes}

In a third group of experiments, designed to further explore the effects of C1-COP injections with CTb-S, six rats, trained to walk steadily on a walking belt at $10 \mathrm{~m} / \mathrm{min}$, were chronically instrumented with EMG electrodes in the left BF muscle as well as with subcutaneously placed stimulation electrode near the lateral malleolus of the same paw (Bronsing et al., 2005). During the surgery, four rats received a CTb-S injection into the ipsilateral (left) $\mathrm{C} 1$ COP (CTb-S group), and two rats received a saline injection in the same area (sham group). After a two-day recovery period, performance of the animals on the walking belt was examined. Figure 5 shows examples of raw EMG traces of BF obtained in both experimental groups. Activity of the BF during a step cycle of sham-operated animals consisted of a short burst of activity (BA; darkly shaded areas), followed by a short-lasting silent period (SP; shaded areas) and a second, sustained and less intense period of BF activity (SA; light areas) thought to be related to the extension of the hip during the stance phase (English and Weeks, 1987; Chanaud and Macpherson, 1991). The well recognized on- set of the BA was taken as the beginning of the step cycle, because it reflects the onset of knee flexion at the beginning of the swing phase. In this way, a single step was divided into three phases, which are coded from dark to light gray in Figure 5. This pattern is similar to noninjected rats and can be considered a normal BF EMG pattern (Bronsing et al., 2005).

At first glance, it can be seen that there were no apparent differences in the pattern of BF activity for sham and CTb-Sinjected animals (Fig. 5A,B). The average step duration determined on subsequent days after the surgery also was not significantly different in both situations (Fig. $5 C-E)$. However, note that the distribution of step durations is somewhat more peaked for the CTb-S animals than for the sham animals. This "peakedness" as measured by the sample kurtosis is maximal at postoperative day 4 for the CTb-S animals, suggesting that at this time, the CTb-Sinjected rats demonstrated a more regular gait pattern than the sham-operated ones (Fig. $5 F$ ).

Subsequently, we studied whether the reflex response of the BF during locomotion as resulting from subcutaneous stimulation around the lateral ankle region (see Materials and Methods) was altered. The average duration of steps in which a subcutaneous stimulation was given was only slightly, but significantly, longer compared with nonstimulated steps in both sham $(\Delta \mu=$ $23 \mathrm{~ms} ; p \ll 0.05)$ and CTb-S animals $(\Delta \mu=25 \mathrm{~ms} ; p \ll 0.05)$. The step after a stimulated step showed a small but significant $(p$ $\ll 0.05)$ decrease of the mean step duration in the sham animals only $(\Delta \mu=-18 \mathrm{~ms} ; p \ll 0.05)$. Because these effects of stimulation on the step duration were at best only very marginal, it was concluded that the stimulation itself did not disrupt normal walking behavior.

The size of the BF reflex response to the subcutaneously placed stimulation electrodes was subsequently analyzed. Figure $6 \mathrm{~A}$ shows the BF-EMG trace of a sham-operated animal (case R7) during walking with randomly triggered stimulations of the subcutaneously placed electrodes (stimulated walk). The three phases of the step cycle, again, are coded in gray scale. The same trace is displayed as a rectified signal in Figure $6 \mathrm{~B}$. Within this bout of walking, two stimulation pulses were triggered (red lines). The first fell within the SA phase and hardly resulted in a visible reflex response within the BF EMG. In contrast, the second stimulation (Fig. 6A, asterisk), triggered within the SP phase, produced a marked reflex response in the BF-EMG, which is also shown at a drawn-out time scale (Fig. 6C).

Next, individual steps were isolated and, after standardization (Fig. 6D) and amplitude normalization, sorted into groups of normal steps (Fig. $6 E$ ) and groups of steps that contained a stimulus (Fig. 6F). By averaging the EMG amplitude data for many nonstimulated steps, a "standard" step EMG was calculated for every rat and session (Fig. $6 E$, red line). Per day, the number of normal steps ranged between 722 and $1218(\mu=959.0)$ in the sham group and between 680 and $1881(\mu=1115.8)$ in the $\mathrm{CTb}-\mathrm{S}$ group. The daily number of stimulated steps ranged between 240 and $336(\mu=293.2)$ in the sham group and between 437 and $686(\mu=558.2)$ in the lesion group. Figure $6 E$ shows the superimposed EMG recordings of 513 nonstimulated steps 
(shown in black traces), to produce the average, standardized, BF-EMG (red trace) at day 4 of case R7 (sham operated). The standardized step of every experimental day was taken as a reference for baseline EMG activity on which the reflex responses could be superimposed from the stimulated steps (Fig. $6 \mathrm{~F}$, black traces labeled with yellow dots at the time of the triggered cutaneous stimulation) of the same animal on the same postoperative day. From these two values, the relative reflex magnitude was calculated and plotted against the moment of triggering within the step cycle (Fig. $6 G$, note that the step cycle was divided into $205 \%$ bins). In this way, it was obvious that in the two sham animals, cutaneously induced reflexes are most pronounced during the BA and SP periods, whereas they are suppressed during the SA phase. These observations are in line with a previous study by Bronsing et al. (2005).

Data obtained from the four animals with a C1-COP injection of CTb-S were processed in a similar way and compared with the sham animals. The mean reflex responses per step cycle bin obtained for each animal and for each postoperative day as well as the summed results of these plots [sham (white bars) vs CTb-S (gray bars)] are shown in Figure $7 A$. From these plots, it can be seen that the depth of modulation is considerably reduced in the CTb-S-injected animals. Especially during the initial $20 \%$ of the step cycle, the size of the reflex response was reduced compared with the sham-operated rats. The effect appears less pronounced at postoperative day 3, when the internalized and transported $\mathrm{CTb}-\mathrm{S}$ may not have resulted in adequate degeneration of neurons, and at day 7 , when other adaptive or compensating processes may have begun to take effect. When all reflex responses between postoperative day 3 and 7 were grouped into the three identified phases of BF activity, significant differences between the CTb-treated and sham animals were found for all three main phases. The BF and SP phases showed a diminishing reflex response, whereas a slight increase (i.e., a disinhibition of the normally suppressed reflex during this phase) was observed in the SA phase (Fig. 7B).

We conclude that the CTb-S injection into C1-COP considerably changed the step-dependent modulation of $\mathrm{BF}$ reflexes during locomotion.

\section{Discussion}

\section{Technical considerations}

As pointed out by Bloedel and Bracha (1995), a lesion study does not unequivocally prove the function of that specific part of the CNS; however, it is generally accepted that lesion studies at least can provide important clues or insights for normal functioning. It was the main goal of the present study to obtain such clues on the function of a single cerebellar module. Lesioning such a module with conventional techniques is not feasible, because these modules are very narrow and are distributed over different parts of the cerebellar cortex (Voogd et al., 2003; Sugihara and Shinoda, 2004; Pijpers et al., 2005). The Purkinje cells of our targeted module, i.e., the $\mathrm{C} 1$-hindlimb module in $\mathrm{COP}$, receive their climbing fiber input from the caudolateral part of the vfDAO, which is known to possess receptive fields related to the ipsilateral hindlimb (Atkins and Apps, 1997; Pardoe and Apps, 2002; Voogd et al., 2003). Olivary neurons of this part of the vfDAO also distribute climbing fibers to the $\mathrm{C} 1$ and $\mathrm{C} 3$ zones in the anterior lobe (Pijpers et al., 2005). Furthermore, many climbing fibers collateralize to reach both the COP as well as the anterior lobe parts of this module (Voogd et al., 2003; Pijpers et al., 2006). Purkinje cells of all these related cortical regions project to the medial part of the AIN (Apps and Garwicz, 2005; Pijpers et al., 2005). Hence, these cortical zones together with the caudolateral part of vfDAO and the medial AIN are considered to constitute the $\mathrm{C} 1 / \mathrm{C} 3$ hindlimb module. Our results depend critically on the effectiveness of the suicide tracer to impair all these regions without affecting adjacent cerebellar modules. Llewellyn-Smith et al. (1999, 2000) injected $\mathrm{CTb}$-saporin into either the superior cervical ganglion or the facial nerve and compared its neurotoxic effects on sympa- 
A

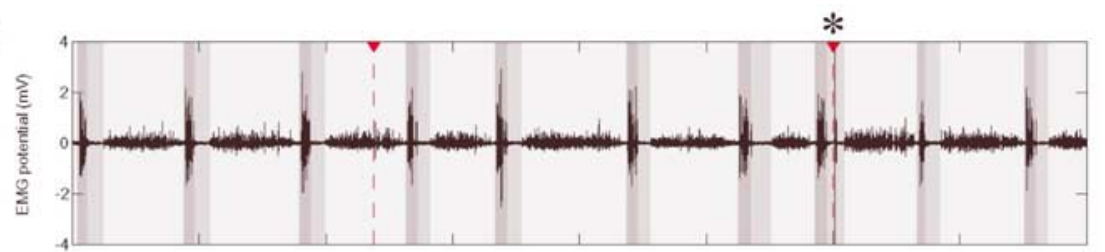

B

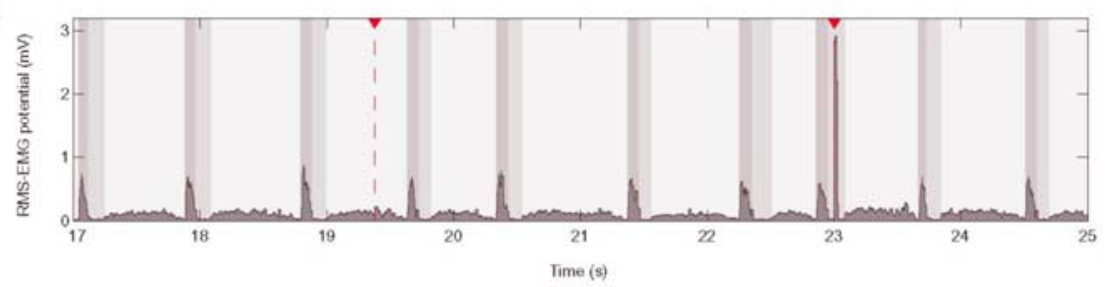

C

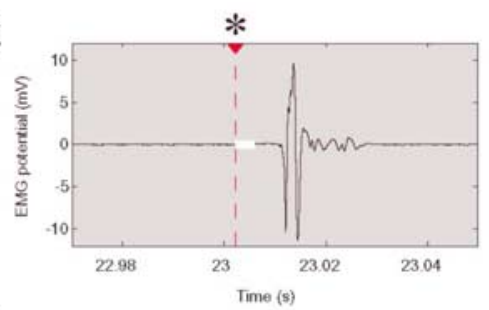

D

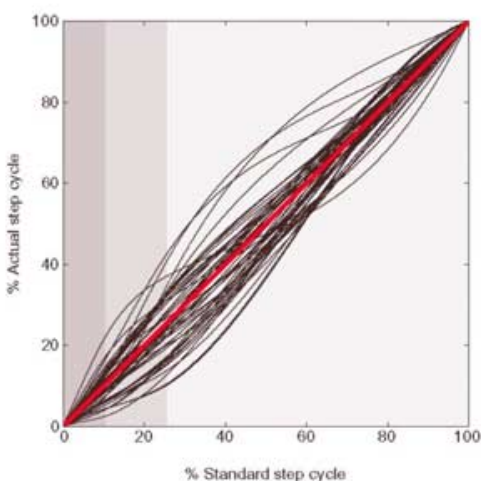

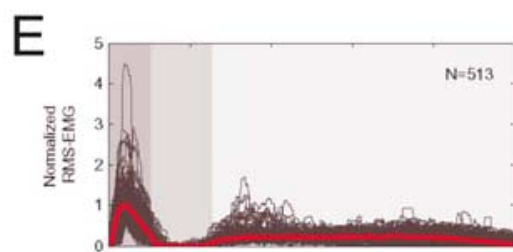

$\mathrm{F}$

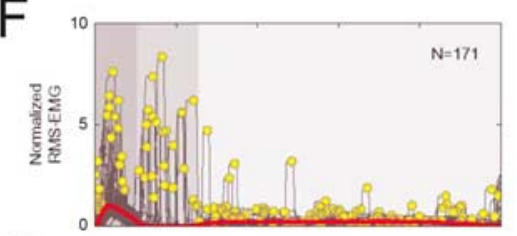

G

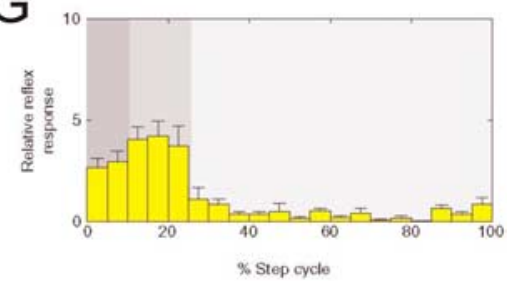

Figure 6. Step standardization and analysis of relative reflex responses derived from BF EMG activity in the left hindlimb during stimulated walking. $\boldsymbol{A}$, Trace of recorded EMG activity (in millivolts) during a session with random triggering, indicated by the red dashed lines, of the subcutaneously placed stimulating electrodes. $\boldsymbol{B}$, EMG amplitude (in millivolts) computed by RMS rectification using a $20 \mathrm{~ms}$ window. $C$, Enlargement of the reflex response as part of the EMG potential (in millivolts) derived from the second stimulus in $\boldsymbol{A}$ (asterisk). The white bar shows the masked area containing the stimulus artifact. $\boldsymbol{D}$, Step standardization by time rescaling of 50 example steps (randomly drawn). The relative time axis of each step (black lines) was smoothly rescaled to fit the standard step phase lengths (gray shaded areas). Time is compressed where slopes exceed 1 and stretched where slopes are smaller than 1 . The red line indicates no rescaling. $\boldsymbol{E}, \boldsymbol{F}$, Normalized RMS-EMG amplitude data were divided into groups of normal, nonstimulated steps $(\boldsymbol{E})$ and of stimulated steps ( $\boldsymbol{F}$; different scale). The red trace reflects the EMG amplitude data of the normal step group, which was averaged per rat for each day. The yellow dots in $\boldsymbol{F}$ represent the reflex magnitude as the height of the normalized EMG amplitude $15 \mathrm{~ms}$ after a stimulus. G, Relative reflex responses averaged in 5\% step cycle bins. Net reflex magnitudes \pm SEM were calculated by subtracting the corresponding value of the average step cycle EMG activity from the reflex magnitude observed in $\boldsymbol{F}$.

thetic preganglionic or motor neurons with injections of unconjugated saporin. They concluded that, because CTb binds to GM1 ganglioside, the conjugate would be effective in the specific elimination of all neurons that express this molecule in their membranes.

Previous anatomical tracing studies within the cerebellar modular system already demonstrated that $\mathrm{CTb}$ is readily transported throughout its different constituents (Voogd et al., 2003; Pijpers et al., 2006; Ruigrok and Apps, 2007). The present study shows that CTb-S injection not only results in degeneration of Purkinje cells located at and directly surrounding the injection site, but also of the mossy and climbing fiber sources that reach the injected area. As a result of degeneration of the parent soma, the axonal branches of climbing as well as mossy fiber afferents to other cerebellar regions will degenerate as well. Because especially the climbing fiber deafferentation of Purkinje cells is expected to severely affect their functional characteristics (Batini et al., 1985), a single injection of CTb-S in C1COP is expected to impair a major part of a functional modular entity. In this respect it is important to realize that recurrent corticonucleocortical loops are also operative within these modules. The present data provide evidence that at least some of the nucleocortical neurons within the AIN, in addition to the cortical axon to the C1-COP, also possess a projection axon directed to the magnocellular red nucleus and possibly beyond. This suggests that the $\mathrm{CTb}-\mathrm{S}$ injections in C1-COP not only result in impaired activity of AIN neurons, but that a specific part of its output will be completely abolished.

Hence, we conclude that a CTb-S injection that is restricted to particular cortical aspect of a module results in a major impairment of large parts of this module, thereby resulting in a modularly restricted impairment of its function, and as such provides a new and promising tool to investigate the isolated function of these modules in behavioral paradigms such as, e.g., locomotion.

\section{No overt behavioral effects after impairment of the C1-hindlimb module} Problems with coordination of locomotion, often referred to as gait ataxia, are usually one of the first and major signs of cerebellar disease (Dow and Moruzzi, 1958). Therefore, it was quite unexpected that none of the animals in which a major part of the C1-hindlimb module was impaired showed any overt signs of walking disability. Also, with the more sophisticated analysis using the ladder rungwalking test, no obvious problems with skilled stepping came to light. This was in marked contrast to the effects of motor cortex lesions, which did result in frequent misplacements of the contralateral hindpaw. Hence, cerebellar lesions do not necessarily have to lead to easily recognizable deficits, especially if they specifically target single modules. Selective lesions of the magnocellular red nucleus (mRN) also do not seem to lead to major disturbances in locomotion (Muir and Whishaw, 2000). Because the mRN constitutes one of the major output sources of the AIN (Teune et al., 2000; Ruigrok, 2004), this observation is in good agreement with the present findings. We, therefore, suggest that the C1-hindlimb module plays a very selective role in motor coordination and presume that the typical symptoms of ataxia will only result from disruption of other modules or, potentially, of multiple modules. Note that our C1 
impairments seemed to result in a more even pattern of gait, whereas cerebellar ataxia is characterized by increased variation in stepping parameters (Ilg et al., 2007).

\section{C1-hindlimb module involved in reflex modulation}

At the level of the spinal cord, central pattern generators are thought to produce the basic walking rhythm (Juvin et al., 2005). However, because walking has to be adapted constantly to functionally relevant environmental requirements, this behavior cannot be stereotyped, and sensory modalities are continuously used to finetune individual steps. To a large extent, this occurs unconsciously by means of phase- and task-dependent modulation of reflexes (Forssberg et al., 1975; Duysens et al., 1990; Pearson, 1995). For instance, Drew and Rossignol (1987) already showed in cats that during the swing phase, cutaneous receptors correct and modify locomotive limb movements. In a more recent study, Bronsing et al. (2005)

showed that modulation of cutaneously induced reflexes can also be demonstrated in EMG recordings of BF and gastrocnemius muscles in walking rats. In line with this observation, input and output pathways to and from the paravermal parts of the cerebellum of the cat seem to be modulated during locomotion (Arshavsky et al., 1986; Apps and Lee, 1999). Therefore, the cerebellar paravermis, which involves the C-modules, would seem to be an ideal candidate to monitor and adjust these reflexes in a learning-dependent way, especially because they have been implicated to be involved in controlling withdrawal reflexes (Kolb et al., 1997; Bracha et al., 1999; Apps and Garwicz, 2005). Indeed, the present results show that a selective impairment of the hindlimb-related $\mathrm{C} 1$ zone reduces the depth of modulation of these reflexes. It is to be expected that such a reduced modulation will only really hamper the animal when it is tested for their specific requirements and may hardly be noted during normal walking. In this respect, it was interesting to see that the impaired rats walked with more evenly timed paces, suggesting that it did not want to or could not rely on reflexes that may be specifically operative during a more haphazard gait pattern.

\section{Implications for modular cerebellar functioning}

The cerebellar influence on reflex gain has been known for some time (MacKay and Murphy, 1979; Kolb et al., 1997). However, in recent decades the role of the cerebellum has been studied most extensively in relation to the adaptation of compensatory eye movements (Blazquez et al., 2004) and eye blink paradigms (De Zeeuw and Yeo, 2005). Especially studies on eye blink conditioning have demonstrated that the cerebellum, apart from facilitating the newly acquired response, is also involved in controlling the timing and amplitude of the conditioned reflex, thereby aiding in both motor learning and motor performance (Koekkoek et al., 2003). With respect to cerebellar involvement to voluntary movements and locomotion, we have only just begun to appreciate and pinpoint more precisely its modulatory actions (Bracha et al., 1999; Apps and Garwicz, 2005). For example, recent studies with human cerebellar patients indicated that the cerebellum is
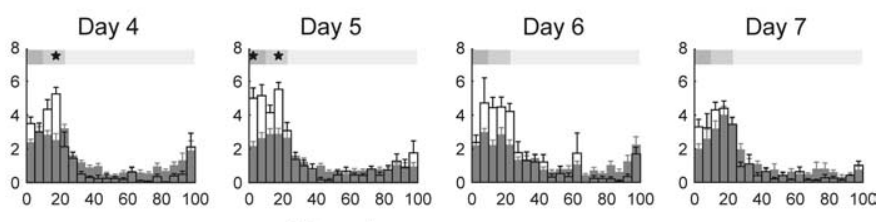

$\%$ Step cycle

Day 3-7

$\star$

$\star$

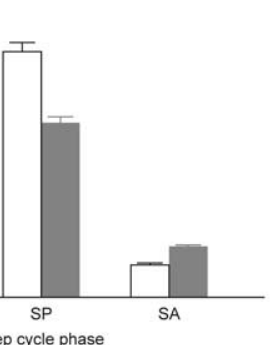

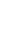

Figure 7. Comparison of relative reflex magnitudes between the sham (white bars) and (Tb-S (gray bars) group. $A$, Relative eflex responses \pm SEM averaged in $5 \%$ step cycle bins as a function of the step cycle for postoperative days $3-7$. $\boldsymbol{B}$, Relative reflex responses averaged in the three step cycle phases with data pooled for all postoperative days. Stars represent significant differences $(\boldsymbol{A}, p<0.0005 ; \boldsymbol{B}, p<0.015)$.

involved in the timing of withdrawal reflexes (Kolb et al., 2007). Other studies have indicated that the cerebellum is implicated in operant conditioning of the H-reflex (Chen and Wolpaw, 2005). Here, we propose that during locomotion, the C1-hindlimb module can fulfill a similar role by continuously and adaptively adjusting peripherally evoked reflexes to apply them optimally in various behavioral tasks. Such adaptive control will ensure that changing functional demands but also changing characteristics of the locomotor apparatus or of ever-changing environmental requirements will constantly result in the best possible use of reflexes.

\section{References}

Apps R, Garwicz M (2005) Anatomical and physiological foundations of cerebellar information processing. Nat Rev Neurosci 6:297-311.

Apps R, Lee S (1999) Gating of transmission in climbing fibre paths to cerebellar cortical C1 and C3 zones in the rostral paramedian lobule during locomotion in the cat. J Physiol (Lond) 516:875-883.

Arshavsky YI, Gelfand IM, Orlovsky GN (1986) Cerebellum and rhythmical movements. Berlin: Springer.

Atkins MJ, Apps R (1997) Somatotopical organisation within the climbing fibre projection to the paramedian lobule and copula pyramidis of the rat cerebellum. J Comp Neurol 389:249-263.

Batini C, Billard JM, Daniel H (1985) Long term modification of cerebellar inhibition after inferior olive degeneration. Exp Brain Res 59:404-409.

Blazquez PM, Hirata Y, Highstein SM (2004) The vestibulo-ocular reflex as a model system for motor learning: what is the role of the cerebellum? Cerebellum 3:188-192.

Bloedel JR, Bracha V (1995) On the cerebellum, cutaneomuscular reflexes, movement control and the elusive engrams of memory. Behav Brain Res 68:1-44.

Bracha V, Kolb FP, Irwin KB, Bloedel JR (1999) Inactivation of interposed nuclei in the cat: classically conditioned withdrawal reflexes, voluntary limb movements and the action primitive hypothesis. Exp Brain Res 126:77-92.

Bronsing R, van der Burg J, Ruigrok TJ (2005) Modulation of cutaneous reflexes in hindlimb muscles during locomotion in the freely walking rat: a model for studying cerebellar involvement in the adaptive control of reflexes during rhythmic movements. Prog Brain Res 148:243-257.

Chanaud CM, Macpherson JM (1991) Functionally complex muscles of the cat hindlimb. III. Differential activation within biceps femoris during postural perturbations. Exp Brain Res 85:271-280. 
Chen XY, Wolpaw JR (2005) Ablation of cerebellar nuclei prevents H-reflex down-conditioning in rats. Learn Mem 12:248-254.

De Zeeuw CI, Yeo CH (2005) Time and tide in cerebellar memory formation. Curr Opin Neurobiol 15:667-674.

Desclin JC (1974) Histological evidence supporting the inferior olive as the major source of cerebellar climbing fibers in the rat. Brain Res 77:365-388.

Dow RS, Moruzzi G (1958) The physiology and pathology of the cerebellum. Minneapolis: University of Minnesota.

Drew T, Rossignol S (1987) A kinematic and electromyographic study of cutaneous reflexes evoked from the forelimb of unrestrained walking cats. J Neurophysiol 57:1160-1184.

Duysens J, Trippel M, Horstmann GA, Dietz V (1990) Gating and reversal of reflexes in ankle muscles during human walking. Exp Brain Res 82:351-358.

English AW, Weeks OI (1987) An anatomical and functional analysis of cat biceps femoris and semitendinosus muscles. J Morphol 191:161-175.

Forssberg H, Grillner S, Rossignol S (1975) Phase dependent reflex reversal during walking in chronic spinal cats. Brain Res 85:103-107.

Gravel C, Hawkes R (1990) Parasagittal organization of the rat cerebellar cortex: direct comparison of Purkinje cell compartments and the organization of the spinocerebellar projection. J Comp Neurol 291:79-102.

Haasdijk ED, Vlug A, Mulder MT, Jaarsma D (2002) Increased apolipoprotein E expression correlates with the onset of neuronal degeneration in the spinal cord of G93A-SOD1 mice. Neurosci Lett 335:29-33.

Haroian AJ, Massopust LC, Young PA (1981) Cerebellothalamic projections in the rat: an autoradiographic and degeneration study. J Comp Neurol 197:217-236.

Ilg W, Golla H, Thier P, Giese MA (2007) Specific influences of cerebellar dysfunctions on gait. Brain 130:786-798.

Juvin L, Simmers J, Morin D (2005) Propriospinal circuitry underlying interlimb coordination in mammalian quadrupedal locomotion. J Neurosci 25:6025-6035.

Koekkoek SK, Hulscher HC, Dortland BR, Hensbroek RA, Elgersma Y, Ruigrok TJ, De Zeeuw CI (2003) Cerebellar LTD and learningdependent timing of conditioned eyelid responses. Science 301:1736-1739.

Kolb FP, Irwin KB, Bloedel JR, Bracha V (1997) Conditioned and unconditioned forelimb reflex systems in the cat: involvement of the intermediate cerebellum. Exp Brain Res 114:255-270.

Kolb TF, Lachauer S, Schoch B, Gerwig M, Timmann D, Kolb FP (2007) Comparison of the electrically evoked leg withdrawal reflex in cerebellar patients and healthy controls. Exp Brain Res 177:493-508.

Llewellyn-Smith IJ, Martin CL, Arnolda LF, Minson JB (1999) Retrogradely transported CTB-saporin kills sympathetic preganglionic neurons. NeuroReport 10:307-312.

Llewellyn-Smith IJ, Martin CL, Arnolda LF, Minson JB (2000) Tracer-toxins: cholera toxin B-saporin as a model. J Neurosci Methods 103:83-90.

Luppi P-H, Aston-Jones G, Akaoka H, Chouvet G, Jouvet M (1995) Afferent projections to the rat locus coeruleus demonstrated by retrograde and anterograde tracing with cholera-toxin B subunit and Phaseolus vulgaris leucoagglutinin. Neuroscience 65:119-160.

MacKay WA, Murphy JT (1979) Cerebellar modulation of reflex gain. Prog Neurobiol 13:361-417.

Metz GA, Whishaw IQ (2002) Cortical and subcortical lesions impair skilled walking in the ladder rung walking test: a new task to evaluate foreand hindlimb stepping, placing, and co-ordination. J Neurosci Methods 115:169-179.

Muir GD, Whishaw IQ (2000) Red nucleus lesions impair overground locomotion in rats: a kinetic analysis. Eur J Neurosci 12:1113-1122.

Pardoe J, Apps R (2002) Structure-function relations of two somatotopi- cally corresponding regions of the rat cerebellar cortex: olivo-corticonuclear connections. Cerebellum 1:165-184.

Pearson (1995) Proprioceptive regulation of locomotion. Curr Opin Neurobiol 5:786-791.

Pijpers A, Ruigrok TJ (2006) Organization of pontocerebellar projections to identified climbing fiber zones in the rat. J Comp Neurol 496:513-528.

Pijpers A, Voogd J, Ruigrok TJ (2005) Topography of olivo-cortico-nuclear modules in the intermediate cerebellum of the rat. J Comp Neurol 492:193-213.

Pijpers A, Apps R, Pardoe J, Voogd J, Ruigrok TJ (2006) Precise spatial relationships between mossy fibers and climbing fibers in rat cerebellar cortical zones. J Neurosci 26:12067-12080.

Provini L, Marcotti W, Morara S, Rosina A (1998) Somatotopic nucleocortical projections to the multiple somatosensory cerebellar maps. Neuroscience 83:1085-1104.

Ruigrok TJ, Apps R (2007) A light microscope-based double retrograde tracer strategy to chart central neuronal connections. Nat Protoc 2:1869-1878.

Ruigrok TJH (1997) Cerebellar nuclei: the olivary connection. In: The cerebellum: from structure to control (De Zeeuw CI, Strata P, Voogd J, eds), pp 162-197. Amsterdam: Elsevier Science.

Ruigrok TJH (2004) Precerebellar nuclei and red nucleus. In: The rat nervous system, Ed 3 (Paxinos G, ed), pp 167-204. San Diego: Elsevier Academic.

Ruigrok TJH, Teune TM, van der Burg J, Sabel-Goedknegt H (1995) A retrograde double labeling technique for light microscopy. A combination of axonal transport of cholera toxin B-subunit and a gold-lectin conjugate. J Neurosci Methods 61:127-138.

Sugihara I, Shinoda Y (2004) Molecular, topographic, and functional organization of the cerebellar cortex: a study with combined aldolase $\mathrm{C}$ and olivocerebellar labeling. J Neurosci 24:8771-8785.

Sugihara I, Ebata S, Shinoda Y (2004) Functional compartmentalization in the flocculus and the ventral dentate and dorsal group y nuclei: an analysis of single olivocerebellar axonal morphology. J Comp Neurol 470:113-133.

Teune TM, van der Burg J, van der Moer J, Voogd J, Ruigrok TJH (2000) Topography of cerebellar nuclear projections to the brain stem in the rat. In: Cerebellar modules: molecules, morphology and function (Gerrits NM, Ruigrok TJH, De Zeeuw CI, eds), pp 141-172. Amsterdam: Elsevier Science.

Tolbert DL, Bantli H, Bloedel JR (1978) Multiple branching of cerebellar efferent projections in cats. Exp Brain Res 31:305-316.

Voogd J, Glickstein M (1998) The anatomy of the cerebellum. Trends Neurosci 2:305-371.

Voogd J, Ruigrok TJ (2004) The organization of the corticonuclear and olivocerebellar climbing fiber projections to the rat cerebellar vermis: the congruence of projection zones and the zebrin pattern. J Neurocytol 33:5-21.

Voogd J, Pardoe J, Ruigrok TJ, Apps R (2003) The distribution of climbing and mossy fiber collateral branches from the copula pyramidis and the paramedian lobule: congruence of climbing fiber cortical zones and the pattern of zebrin banding within the rat cerebellum. J Neurosci 23:4645-4656.

Welsh JP, Harvey JA (1991) Pavlovian conditioning in the rabbit during inactivation of the interpositus nucleus. J Physiol (Lond) 444:459-480.

Welsh JP, Harvey JA (1998) Acute inactivation of the inferior olive blocks associative learning. Eur J Neurosci 10:3321-3332.

Woolf CJ, Swett JE (1984) The cutaneous contribution to the hamstring flexor reflex in the rat: an electrophysiological and anatomical study. Brain Res 303:299-312. 